\title{
LEGged 3D PRINTED MOBILE ROBOT
}

\author{
Petar Ćurković, Luka Mišković \& David Šarančić
}
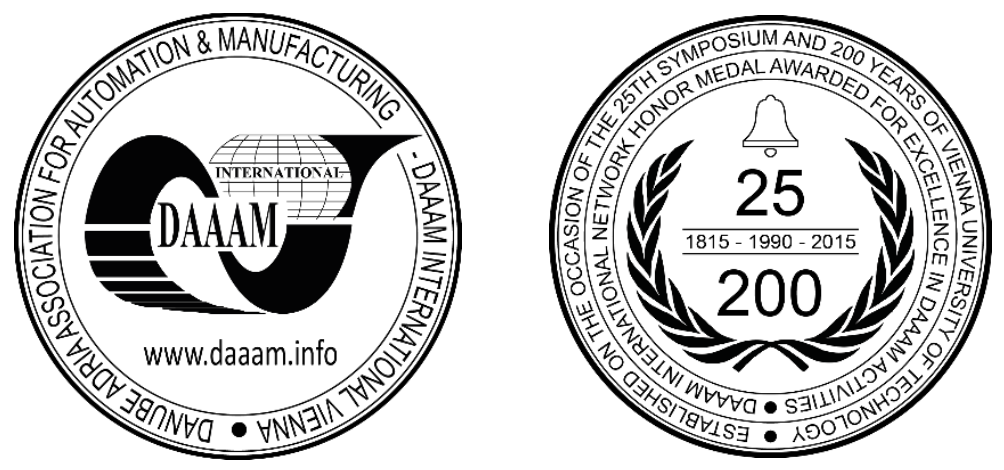

This Publication has to be referred as: Curkovic, P[etar]; Miskovic, L[uka] \& Sarancic, D[avid] (2018). Legged 3D Printed Mobile Robot, Proceedings of the 29th DAAAM International Symposium, pp.0394-0399, B. Katalinic (Ed.), Published by DAAAM International, ISBN 978-3-902734-20-4, ISSN 1726-9679, Vienna, Austria DOI: $10.2507 / 29$ th.daaam.proceedings.057

\begin{abstract}
This paper presents redesign and improvement of the open source four-legged 8 DOF mobile robot platform Aracna. This robot is developed for educational and research purposes, mainly to test different AI-powered methods for gait generation and optimization. We have modified the complete electrical setup of the platform by implementing state of the art electronics. Programming and interaction of programmer and robot is made easier and more intuitive. Weight of the robot is reduced by $0,2 \mathrm{~kg}$. Complete physical setup of the robot is redesigned and optimized to take the new electronics. The robot is printed as an assembly using Objet's VeroBlue material. First experiments demonstrate better performance in terms of the level of autonomy compared to original version. Additionally, range of motion of legs is increased, enabling the robot to move faster.
\end{abstract}

Keywords: mobile robot; legged motion; gait generation; artificial intelligence; additive technologies

\section{Introduction}

Mobile robots are applicable in variety of applications, especially in hazardous environments. A mobile robot requires propelling mechanisms that realize motion. Their motion can be effectuated by diverse means of locomotion. Legged locomotion is the most suitable for harsh terrains due to its ability to overcome all kinds of obstacles. Most legged mobile robots are made by imitating animals and humans. This approach of implementing knowledge obtained from observing nature into technical systems is called biomimetics [1].

In recent years, we are encountering more complex problems and mechanisms. Their movement becomes nonintuitive; accordingly, it requires more sophisticated control and learning algorithms. In such demanding applications, artificial intelligence algorithms surpass performance of human engineers [2],[3],[4]. Having that in mind, we chose to reconstruct quadruped robotic platform with counter-intuitive gait mechanism. This platform is unique because all the actuators are located in the body of the robot, and none of them is in the robot's legs. Specifically, each of the four legs is controlled by separate four-bar linkage mechanism. This design causes unconventional kinematics, thus providing challenging platform for gait-learning algorithms. Evolutionary algorithms will be applied to developed platform in order to generate optimized gaits.

The main goal of this project is adaptation and adjustment of existing robotic platform, developed at Cornell University [5],[6]. Improvements are made in the aspects of design, electronic circuitry, increased autonomy, gait generation and mass reduction. 
The model of the robot that was designed and thoroughly analyzed through kinematic and FEM simulation is presented in fig. 1. right. The physical model of the robot with all the parts assembled in a fully functional robotic platform is shown in fig. 1 left.
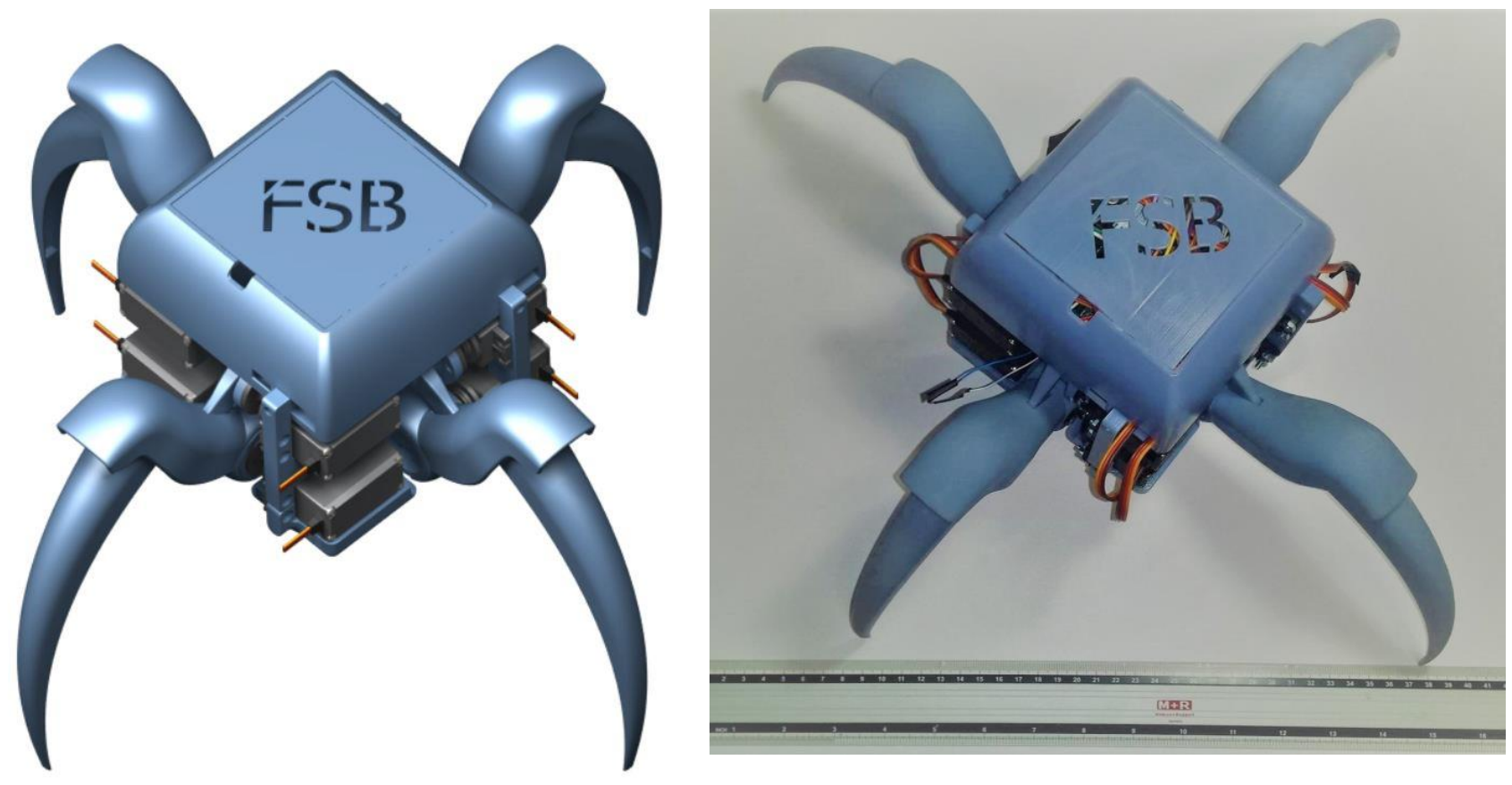

Fig. 1. CAD model of the robot (left). Physical model of the robot (right)

\section{Enhancement}

Originally, platform weighed $1.29 \mathrm{~kg}$ and was printed out of 11 parts. Our team managed to reduce its mass substantially, for approximately $200 \mathrm{~g}$. Reduction in mass is accomplished by usage of material with lower density, as well as optimized design. The new design is distinguished from previous in the form of a bigger leg span. This new model consists of 19 separately printed parts. Some of the initial parts are split into smaller pieces, hence enabling straightforward assembly and possibility to supplant damaged or modified components. Also, this modification excludes potential printing errors, such as bonding to a rigid joint [7].

Considering electronics, significant changes were made in order to accomplish greater autonomy and efficiency of the robot. Selection of lower nominal voltage servomotors enabled application of two serial-connected batteries instead of three, contributing to space saving. Although using lower torque servomotors, performance of the robot is not diminished since the robot is lighter. Likewise, battery capacity is notably increased, consequently ensuring longer lifespan by $74 \%$. Table 1 . shows main differences between the original Aracna platform, and the upgraded version presented in this paper.

\begin{tabular}{|c|c|c|}
\hline Characteristic & Original robot & Enhanced robot \\
\hline Actuators & AX 18-A & TowerPro MG995 \\
\hline Power supply & 3S 11.1 V 2000 mAh LiPo & 2S 8.4 V 3500 mAh Li-Ion \\
\hline Controller & ArbotiX Robocontroller & Arduino Uno \\
\hline Weight & $1.29 \mathrm{~kg}$ & $1.09 \mathrm{~kg}$ \\
\hline Leg span & $48 \mathrm{~cm}$ & $52 \mathrm{~cm}$ \\
\hline Printed parts & 11 & 19 \\
\hline Lifespan & $6.82 \mathrm{~min}$ & $26.25 \mathrm{~min}$ \\
\hline
\end{tabular}

Table 1. Comparison between original and enhanced robot

\section{Mechanical aspects}

To achieve optimized design, that is strong enough to withstand exerted force upon its legs, and on the other hand minimizes mass, FEM analysis is used [8]. Critical spots of this robot are its lower legs, since they are exposed to the highest stress. After iterative simulations with suitable material properties, optimal thickness of the lower legs was adopted. Analysis is conducted using tetrahedron finite elements with twelve degrees of freedom. 
These finite elements can only describe linear displacements and constant fields of stress and strain. FEM results showed maximum elongation of only $1 \mathrm{~mm}$ under peak equivalent Von Mises stress of $5 \mathrm{~N} / \mathrm{mm}^{2}$, which appears due to robot's mass. That is more than satisfying for VeroBlue material. This material is very suitable for such conditions, owing to the fact that its elongation on break is around 20\%. Figure 2. shows FEM analysis on the lower leg, note that stress legend is displayed in $\mathrm{N} / \mathrm{m}^{2}$.
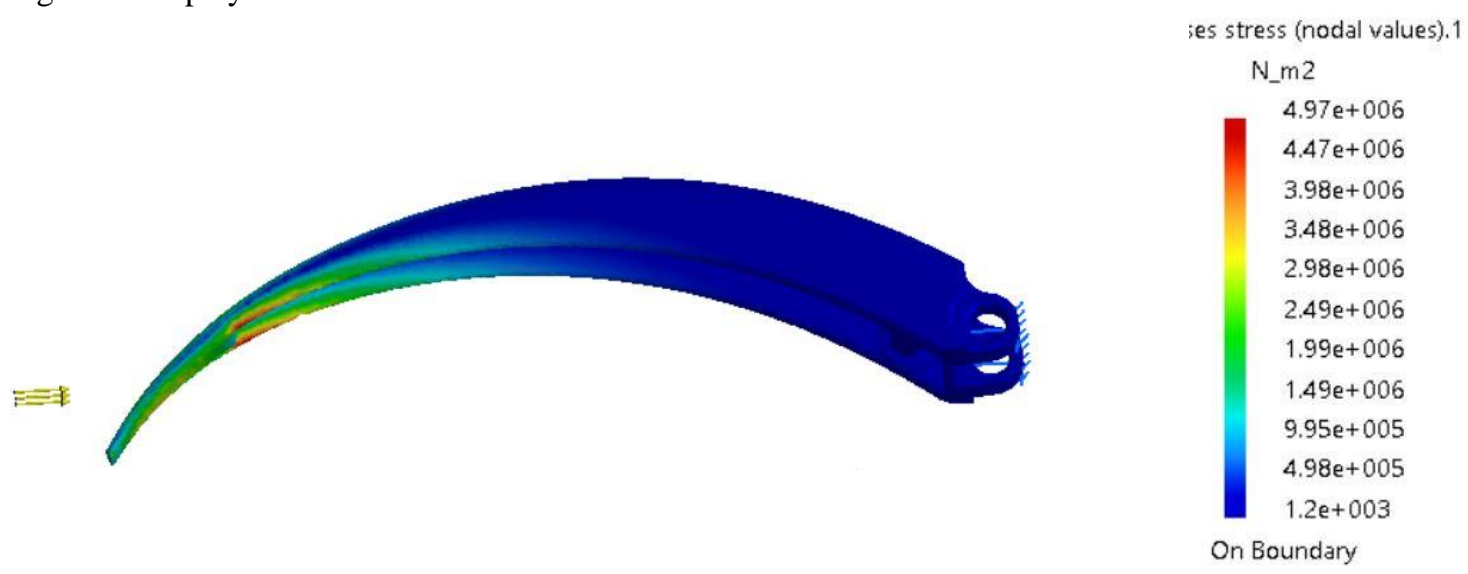

Fig. 2. Lower leg FEM analysis

When it comes to rapid prototyping, used device is Objet30 printer by Stratasys. Table 2. shows weight, cost, in Croatian Kuna, and printing time for particular parts.

\begin{tabular}{|c|c|c|c|}
\hline Part & Weight & Cost of one sample & Print duration of one sample \\
\hline & $66 \mathrm{~g}$ & $330 \mathrm{kn}$ & $6 \mathrm{~h} \mathrm{i} 30 \mathrm{~min}$ \\
\hline & $4 \times 6 \mathrm{~g}$ & $30 \mathrm{kn}$ & $60 \mathrm{~min}$ \\
\hline & $4 \times 4 \mathrm{~g}$ & $20 \mathrm{kn}$ & $35 \mathrm{~min}$ \\
\hline & & & $30 \mathrm{~min}$ \\
\hline & & $15 \mathrm{kn}$ & \\
\hline & $90 \mathrm{~g}$ & $450 \mathrm{kn}$ & $10 \mathrm{~h}$ \\
\hline & & & $45 \mathrm{~min}$ \\
\hline & & & \\
\hline & & & \\
\hline
\end{tabular}

Table 2. List of printed parts

\section{Leg mechanism and kinematics}

Each of four legs is designed in such manner that it consists of two four-bar linkages which moves lower and upper leg. Four-bar linkage is one of the simplest crank-rocker mechanisms [9]. One mechanism contains one degree of freedom which is derived from expression below 
$w=3 \cdot(n-1)-2 \cdot p_{1}-p_{2}=3 \cdot(4-1)-2 \cdot 4=1$

where $n$ is the total number of parts in mechanism, $p_{1}$ is the number of kinematic pairs with one degree of freedom and $p_{2}$ is the number of kinematic pairs with two degrees of freedom. Figure 3. represents working space of the leg with its marginal angles. It is obvious that span of the upper leg settles around $19.5^{\circ}$, whereas lower leg can be turned for $38.5^{\circ}$.

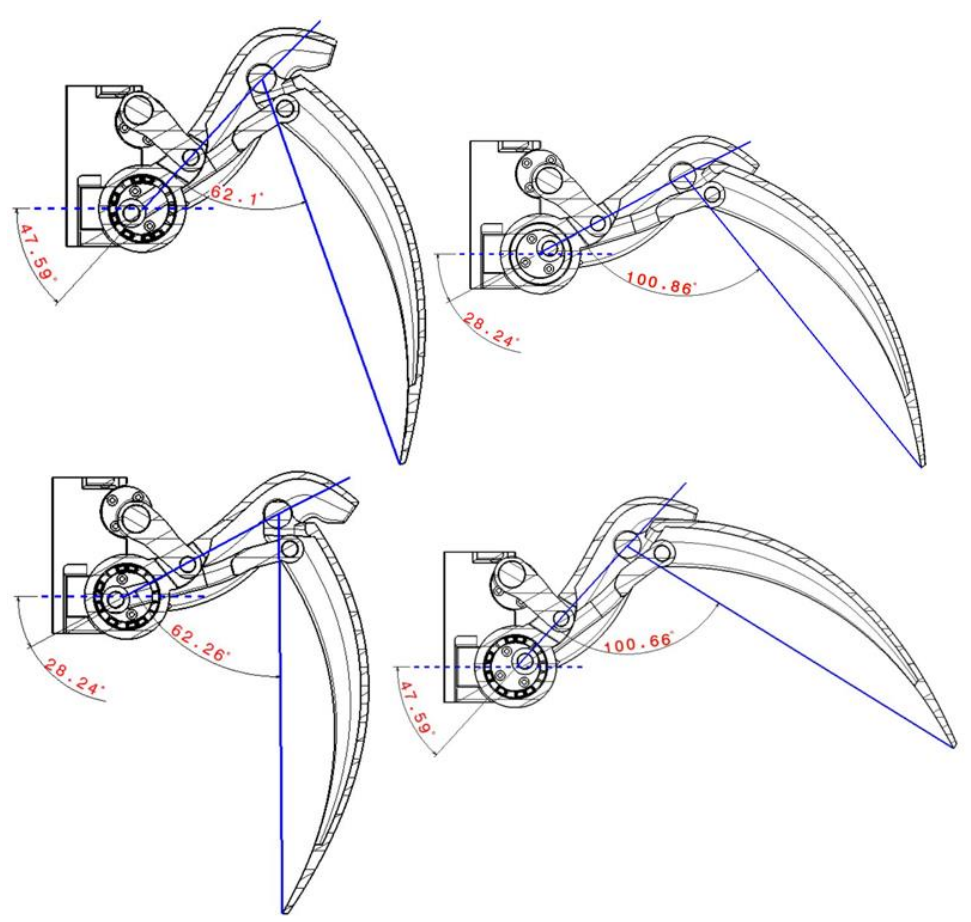

Fig. 3. Range of motion of lower and upper leg

It is very challenging to coordinate four legs, for that reason gait had to be carefully planned. For this leg arrangement, optimal sequence of leg movement means that two legs lift in the same time, while other two legs generate a push [10],[11].

\section{Electronics and programming}

Battery life time is calculated taking into consideration the maximum current draw of servomotors. Idle current of a servomotor is measured at around $450 \mathrm{~mA}$, which is shown in fig. 4.

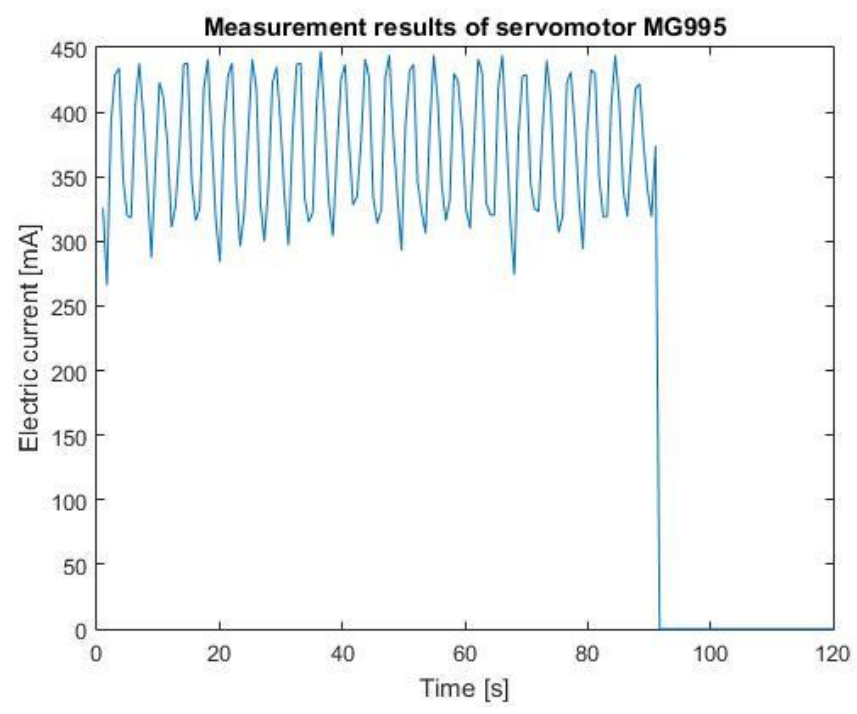

Fig. 4. Idle current draw of servomotor 
Fully loaded motor operating at $1.1 \mathrm{Nm}$ draws around $1 \mathrm{~A}$ of current. Considering measurement results and supposing that all eight servomotors are working constantly at maximum load it is easy to calculate battery life time by the following equation: $t=\frac{C}{8 \cdot I}=\frac{3500 \mathrm{mAh}}{8 \cdot 1000 \mathrm{~mA}}=0.4375 \mathrm{~h}=26.25 \mathrm{~min}$. Having met all the necessary preconditions for optimal functioning, it is possible to research evolutionary algorithms even more in depth.

Interaction of robot and programmer is simplified by using android mobile application, fig. 6. This approach enabled observation of robot's kinematics on a single joint level. Application provides an opportunity to control single servomotor using slider to determine rotating angle. Data gathered with this procedure eases understanding of kinematics and programming of more sophisticated gait learning algorithms. With replacement of the former microprocessor with the Arduino Uno, more user-friendly environment is achieved.

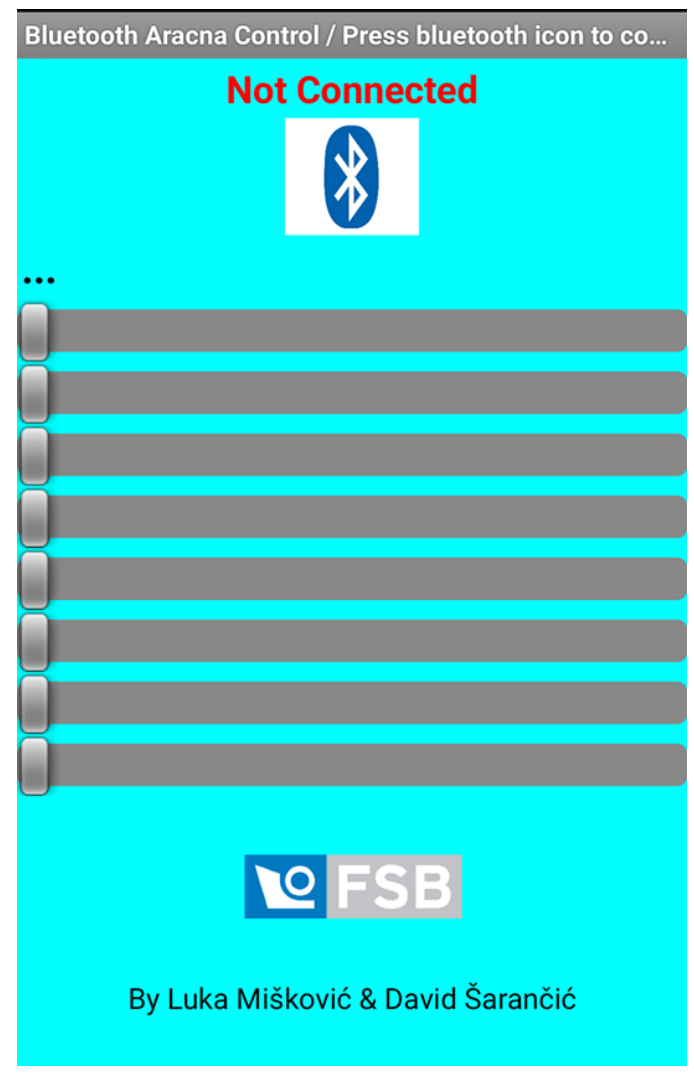

Fig. 5. Bluetooth application

Analyzed data can now be used in path planning and programming of evolutionary algorithms for gait generation [12], [13]. With implementation of such an algorithm, we cannot be certain of learning outcome. Optimal gait is to be generated for the given design and characteristic of the robot. Evolution of the robot is achieved after the repeated application of mutation, recombination and selection operators.

\section{Conclusion}

Efficiency and potential of mobile legged robots is growing considerably, following the development of artificial intelligence. Designed and produced robot represents an excellent platform for future study of artificial intelligence, and evolutionary algorithms in particular. By implementing those algorithms, it is possible to achieve autonomous gait learning. Learning outcome of such an algorithm is partially unpredictable; hence various gaits can be expected. Anticipated gaits are more likely to resemble to starfish movement rather than to spiderlike movement. This mystery can only be answered by further research.

After executing all of above mentioned modifications and improvements, robotic platform is fully adapted and ready for further research. Robot's mass is significantly reduced without tempering with strength of its construction, legs have wider range of motion, and battery can last much longer. The latter gives us more opportunity to conduct lengthy experiments with robot without having to charge it so often, therefore reducing its downtime. This should enable quicker development and testing of primarily evolutionary algorithms for gait generation and path planning. Our future mission is to attach ultrasonic sensors at the top of the robot which will result in higher autonomy, therefore giving the robot more favourable conditions for developing evolutionary algorithms. Sensors also provides mapping of the surroundings which is crucial for orientating in the unknown environment. 


\section{References}

[1] Siegwart, R., Illah, R. Nourbakhsh. (2004). Introduction to Autonomous Mobile Robots. The MIT Press

[2] Lohmann,S., Yosinski,J., Gold, E. , Clune, J., Blum, J., \& Lipson, H. (2013). Aracna: An Open-Source Quadruped Platform for Evolutionary Robotis, ALife Conference

[3] Ćurković, P., Jerbić, B., Stipančić, T. (2009) Swarm-based Approach to Path Planning Using Honey-bees Mating Algorithm and ART Neural Network. Solid state phenomena. 147-149; 74-80

[4] Stipančić, T.,; Jerbić, B., Bučević, A.. Ćurković, P. (2012). Programming an Industrial Robot by Demonstration // Annals of DAAAM for 2012. \& Proceedings of the 23rd International DAAAM Symposium / Katalenić, Branko (ur.). Vienna : DAAAM International, 2012. 15-18

[5] Shen, H. Yosinksi,J., Kormushev,P.,. Caldwell, D. G \& Lipson, H. (2012). Learning Fast Quadruped Robot Gaits with the RL PoWER Spline Parametrization. Cybernetics and Information Technologies, Vol.12, No.3,

[6] Lee, S. Yosinksi, J. Glette, K., Lipson, H. \& Clune,J. (2013). Evolving Gaits for Physical Robots with the HyperNEAT Generative Encoding. European Conference on the Applications of Evolutionary Computation

[7] Dana, M[ilan]; Zetkova, I[vana]; Hanzl, P[avel] \& Hronek, O[ndrej] (2017). Accuracy of Holes Created by 3D Printing (DMLS), Proceedings of the 28th DAAAM International Symposium, pp.0467-0473, B. Katalinic (Ed.), Published by DAAAM International, ISBN 978-3-902734-11-2, ISSN 1726-9679, Vienna, Austria

[8] Munteanu, C[amelia] E[lena]; Cismilianu, A[lexandru] -[Mihai]; Chira, A[linaIoana] \& Baran, D[aniela] (2017). Structural Optimization of Space Components Adapted for 3D Printing, Proceedings of the 28th DAAAM International Symposium, pp.0821-0825, B. Katalinic (Ed.), Published by DAAAM International, ISBN 978-3902734-11-2, ISSN 1726-9679, Vienna, Austria

[9] Lenarcic, J.; Roth, B. (2009). Advances in Robot Kinematics, Springer-Verglag London Limited, Dordrecht, Netherlands

[10] Gonzalez de Santos, P.; Garcia, E.; Estremera, J. (2006). Quadrupedal Locomotion: An introduction to the Control of Four-legged Robots, Springer-Verlag London Limited, Madrid, Spain

[11] Queiroz, C.; Gonçalves, N.; Menezes, P. (2000). A Study on Static Gaits for a Four Legged Robot, International Conference CONTROL'2000, Cambridge, UK

[12] Ćurković, P., Jerbić, B. (2007). Honey-bees optimization algorithm applied to path planning problem. International Journal of Simulation Modelling. VI, 3; 154-165

[13] Ćurković, P.; Jerbić, B.; Stipančić, T. (2013). Coordination of Robots With Overlapping Workspaces Based on Motion Co-Evolution. // International journal of simulation modelling. 12, 1; 27-38 